\title{
A Model for the Viscoelastic Behavior of Rubberlike Polymers Including Entanglement Effects
}

\author{
Robert S. Marvin and Hansjörg Oser
}

(August 22, 1962)

\begin{abstract}
A model representing the mechanical response of a rubberlike polymer is derived, using the same molecular concepts of entropy elasticity and a viscous parameter expressing interactions between polymer molecules employed by Rouse, Bueche, and Zimm in their molecular theories. Since the model developed here represents the mechanical response of a chain, rather than representing the chain itself, it can be modified more easily than these strict molecular theories to include effects due to entanglements between chains which modify the character of the viscosity-molecular weight relationship at a critical molecular weight.

This modification is introduced, the results for both steady-state and transient response functions are calculated, and these results compared with experiment for the "standard" polyisobutylene. The agreement indicates that the same "entanglements," whatever their precise nature, are responsible for the proportionality of viscosity to $M^{3.4}$ for a high molecular weight polymer, the steady-state elastic compliance, and the pseudo-equilibrium compliance at intermediate times or frequencies.
\end{abstract}

\section{Introduction}

Molecular theories of the viscoelastic behavior of rubberlike polymers have been developed in recent years by Rouse [1] ${ }^{1}$, Bueche [2], and Zimm [3]. In these theories the polymer molecule is represented by a model consisting of $N$ segments connected by junctions which permit free rotation. The length of the segments is unspecified, but is assumed long enough to give a Gaussian distribution of end-to-end distances, and hence account for an entropy type elasticity. The interactions between molecules are supposed to be purely viscous (for unvulcanized materials) and these viscous forces are lumped at the junctions as connections between the typical molecule whose motions are analyzed and the average medium, or in the case of the Rouse and Zimm theories the solvent. The Zimm theory includes an approximate treatment of hydrodynamic interaction, that is the coupling of motions along a single chain through the solvent.

Only a simple viscous interaction is included in the basic form of these theories. The entanglement effects which change the character of the viscositymolecular weight relationship at some critical value of molecular weight have been included [4] by treatments which modify the calculated response functions rather than by including them directly in the original model.

We are interested here in representing the mechanical behavior of a high molecular weight, unvulcanized, undiluted polymer in terms of a model whose elements have a clear relationship to molecular quantities. The model we use is basically a phenomenological one, since it represents the mechanical response of a polymer chain rather than representing

1 Figures in brackets indicate the literature refer ences at the end of this paper. the chain itself. It does not seem feasible to include entanglement effects in a model of the polymer molecule, but by dealing with a model representing mechanical response the required modification is relatively simple.

The basic molecular theories have been summarized and compared in some detail by Ferry [5] in his recent book, so there is no need to review them here. The model presented in this paper was discussed briefly in a previous paper [6] and its predictions for steady-state response given. We include here a comparison of the predictions of this model with those of the Rouse theory and the expressions for transient, as well as steady-state, behavior.

\section{Derivation of Model}

We present a derivation of the basic model, relating the elements appearing in a certain form of the response function to molecular concepts, and compare the predictions of this simple model with those of the Rouse theory. We next include a representation of the high frequency limiting shear compliance as a separate element, not connected with the entropy mechanisms dominant at lower frequencies. Finally we modify the model to include the effects of entanglements characteristic of undiluted polymers of high molecular weight.

\subsection{A Model Analogous to the Rouse Theory}

Assume that the complex shear modulus of an unvulcanized polymer, $G^{*}(p)$, where $p$ is a complex frequency $=s+i \omega$, can be represented by $N$ terms of the form $a_{m} p /\left(p+\lambda_{m}\right)$. This sum can be written as the ratio of two polynomials in $p$, both of order $N$, $p P_{N-1}(p) / Q_{N}(p)$, where both $P$ and $Q$ contain nonzero constant terms. This ratio in turn can be 
expressed as a continued fraction by a process of alternate division and inversion, giving for $J^{*}(p)=1 / G^{*}(p)$ :

$$
J^{*}(p)=\alpha_{1}+\frac{1}{\beta_{1} p+\frac{1}{\alpha_{2}+\cdots \frac{1}{\beta_{N} p}}},
$$

where the $\alpha$ 's and $\beta$ 's are functions of the $\lambda_{n}$ 's.

Since the $\alpha$ 's in this expression represent a strain proportional to stress and the $\beta$ 's a stress proportional to rate of strain, this response is represented exactly by the model of figure 1 .

This model represents the mechanical response of an unvulcanized polymer sample, and since the steady-state response is in fact one form of the general response function of a linear system it represents the response to a forcing function (either stress or strain) with any arbitrary time dependence. It is of the same form as the model used to represent a polymer molecule in the Rouse, Bueche, and Zimm theories if we choose the constants as:

$$
\begin{gathered}
1 / \alpha_{1}=1 / \alpha_{2}=\ldots=1 / \alpha_{N}=N k T \\
\beta_{1}=\beta_{2}=\ldots \beta_{N}=\eta / n N,
\end{gathered}
$$

where $N$ is the number of segments in the model representing the polymer molecule, $n$ the number of molecules per cubic centimeter, and $\eta$ the steady flow shear viscosity. Thus we should expect this model to give us the contribution of a single polymer molecule to the shear compliance of a bulk sample. We prefer to use constants that will give directly the response of a bulk sample, requiring the introduction of a factor $n$ as shown in figure 1 .

The response represented by this model is the sum of responses of $1,2,3, \ldots N$ segments acting cooperatively. In this sense it corresponds directly to the normal mode calculation used in the development of the molecular theories. The assignment of constants follows the same molecular concepts used in formulating the model of the molecular theories, the springs representing the entropy-type elastic forces contributed by the segments of a chain, and the dashpots the viscous resistance opposing the motion of a segment.

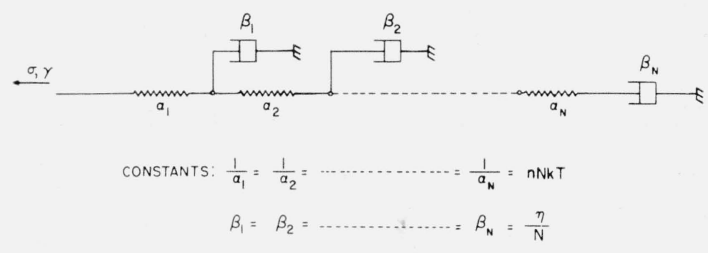

Figure 1. Simple model of mechanical response of polymer chain.

\subsection{Comparison With the Rouse Theory}

The steady-state response of the model of figure 1 is given by: ${ }^{2}$

$J^{*}(p)=1 /(2 N n k T)$

$$
+\left[\frac{1}{p \eta n k T}\left(1+\frac{p \eta}{4 N^{2} n k T}\right)\right]^{1 / 2} \operatorname{ctnh}(N a)
$$

where

$$
\sinh a=\frac{1}{N}\left[\frac{p \eta}{n k T}\left(1+\frac{p \eta}{4 N^{2} n k T}\right)\right]^{1 / 2} .
$$

The Rouse theory gives:

$$
G^{*}(p)=n k T \sum_{j=1}^{N} \frac{p}{p+\lambda_{j}}
$$

with

$$
\lambda_{j}=\frac{2 N(N+2) n k T}{3 \eta} \sin ^{2} \frac{j \pi}{2(N+1)} .
$$

Both expressions depend on $N$, the arbitrary number of segments into which the model of the molecule (or of the response) is divided. Rouse eliminates $N$ from his results by approximations valid for frequencies less than a limit proportional to $N^{2}$. Thus, to obtain expressions valid for all frequencies, we must take the limit as $N$ goes to infinity. This is equivalent to assuming that segments of vanishing length can be treated as kinetic theory springs.

Letting $N$ go to infinity in the model used here eq (2.2) gives:

$$
J^{*}(p)=\sqrt{\frac{1}{p \eta n k T}} \operatorname{ctnh} \sqrt{\frac{p \eta}{n k T}}
$$

$G^{*}(p)$, the reciprocal of $J^{*}(p)$, is given by:

$$
G^{*}(p)=n k T \sqrt{\frac{p \eta}{n k T}} \tanh \sqrt{\frac{p \eta}{n k T}}
$$

or, equivalently, by:

$$
G^{*}(p)=2 n k T \sum_{m=0}^{\infty} \frac{p}{p+\lambda_{m}} \text {, with } \lambda_{m}=\frac{\left(m+\frac{1}{2}\right)^{2} \pi^{2} n k T}{\eta} .
$$

The Rouse expression in this limit is:

$$
G^{*}(p)=n k T \sum_{j=1}^{\infty} \frac{p}{p+\lambda_{j}} ; \lambda_{j}=\frac{j^{2} \pi^{2} n k T}{6 \eta}
$$

or

$$
G^{*}(p)=(n k T / 2)[y \operatorname{ctnh} y-1] ; y=\sqrt{\frac{6 p \eta}{n k T}} .
$$

These two functions are very similar, though not identical. Equation (2.5a), representing the response of our model, can be written as:

2 This solution is obtained by the procedure described in L.A. Pipes, Applied Mathematies for Engineers and Physicists, pp. 265-267 (McGraw-Hill Book Co., New York, 1946). 


$$
G^{*}(p)=(n k T / 2)[x \operatorname{ctnh} x-x / \sinh x] ; x=\sqrt{\frac{4 p \eta}{n k T}} .
$$

This is nearly the same as eq (2.6b), except that $y=(3 / 2)^{\frac{1}{2}} x$. The second term in the brackets of eq $(2.5 \mathrm{c})$ becomes essentially unity for small values of $|p|$, corresponding to that in eq $(2.6 \mathrm{~b})$; for large values of $|p|$ the second term in both expressions is negligible compared to the first.

\subsection{Representation of the Limiting Compliance}

As noted in the last section, the process of going to the limit of infinite $N$ in our model corresponds to taking segments of vanishing length, which results in expressions for $J^{\prime}(\omega)$ vanishing at bigh frequencies as $\omega^{-1 / 2}$. We would not want to use a model which represented the limiting high frequency shear compliance as an entropy-type mechanism, since this would have an unrealistic temperature dependence. Therefore we go to the limit of vanishing segment length and represent this compliance by a separate element in the model.

The model of figure 1 assumes that the junctions between segments are connected to the average medium by purely viscous elements, which show a zero compliance at infinite frequency. In setting up this model we considered only the response associated with rather long chain segments, and these viscous elements represent the resistance to motion experienced by the whole segment in its motion. In addition to this type of response, there also exists the type of compliance characteristic of polymeric glasses, which does not involve long segments of the chains but is connected with forces between individual atoms irrespective of whether or not they are a part of the same polymer molecule. This elastic mechanism will permit a small compliance, negligible at low frequencies in comparison to the entropy response, but becoming dominant as the frequency is increased.

This type of response can be introduced into our model by connecting the viscous elements to ground through a very stiff spring, rather than directly.

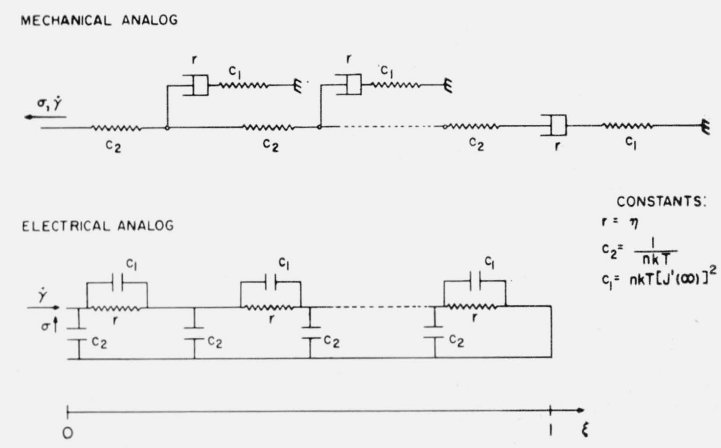

FIGURE 2. Model including limiting high-frequency compliance.
This modification is shown in figure 2, which we represent initially as a model with distributed rather than lumped parameters. The simplest assignment of constants, shown in the figure, is achieved by taking the total length of the model as unity and assigning the constants as shown to represent the response of the bulk sample. $c_{1}$ is evaluated from the calculated response of the model at infinite frequency. In addition to the mechanical model, the electrical analog is also shown, using the system in which voltage corresponds to stress and current to rate of strain.

\subsection{Introduction of Entanglement Effects}

The model as formulated up to this point represents the behavior of a collection of polymer chains in which the motion of any segment or group of segments will be opposed by a viscous force proportional to the length of that segment or group of segments. We know empirically [7] that this simple picture is not adequate to represent the viscous behavior of materials of high molecular weight, and that above some critical molecular weight, characteristic of each polymer, the steady flow viscosity becomes proportional to $M^{3.4}, M$ the molecular weight.

Bueche [8] has developed a theoretical picture in which this effect is attributed to the effects of entanglements between polymer chains, which become effective only when the chains reach some minimum length. Above this critical length he predicts that the viscosity should increase more rapidly than below it, going in the limit of very high molecular weight to an expression proportional to $M^{3.5}$.

We adopt this theoretical picture, using however the empirical exponent of 3.4. We assume that the coordinated motion of any segment or group of segments with a length less than that corresponding to the critical molecular weight will be opposed by a viscous force proportional to the length of the segment or group of segments. When this length exceeds that corresponding to the critical molecular weight, $M_{c}$, we assume that the viscous resistance will still be proportional to length, but the proportionality constant will be much greater, representing the fact that the motion of such a section of the molecule will be opposed by its entanglements with other chains of the sample.

The final model for the response, including entanglement effects, is shown in figure 3. Again this represents a model with distributed parameters. To simplify the final expressions, the length, $l$, of the model is defined as $M / M_{c}$, with the length corresponding to the critical molecular weight taken as unity. $r$ is defined as the viscosity of a polymer of molecular weight equal to the critical molecular weight; $L^{2}$ is defined as $\frac{l^{3.4}-1}{l-1}$ to make the viscosity represented by the model proportional to $M^{3.4}$. $c_{2}$ corresponds to the equilibrium compliance of a 
cross-linked polymer with molecular weight $M_{c} / 2$ between cross links, since this corresponds to the average molecular weight between entanglements.

Although this is really a phenomenological model of response, the constants are simply visualized in terms of our molecular concepts. For motions connected with high-frequency response only short lengths of the polymer chain are involved, and hence only the resistive terms characteristic of small molecules are effective. For lower frequencies, where greater lengths of the chain are involved in cooperative motions, the elements representing entanglement effects become important.

In analyzing the response of this model it will prove useful to employ various approximations valid in certain regions of time or frequency. For this purpose it is necessary to say something about the relative values of the constants appropriate for a model which represents the response of a rubberlike polymer. The constant $c_{2}$, equal to the equilibrium compliance of a vulcanized rubber with molecular weight $M_{c} / 2$, will typically be of the order of $10^{-6} \mathrm{~cm}^{2}$ dyne. $r$ represents the viscosity of a polymer with molecular weight $M_{c}$, so should be within an order of magnitude of $10^{4}$ poise. $l$ is defined as $M / M_{c}$; it must be greater than unity and in most cases where this model would be used it will be considerably greater. In the example considered at the end of this paper it is 60 , yielding a value of $L^{2}=1.9 \times 10^{4} . \quad\left(c_{1} c_{2}\right)^{1 / 2}$ equals the limiting high frequency compliance which is of the order of $10^{-10}$ for all polymers; thus $c_{1}$ will be of the order of $10^{-14}$.

\section{Calculation of Steady-State Response}

The response of a polymer whose mechanical behavior is represented by the model of figure 2 to any arbitrary (shear) stress or strain is found by imposing the given forcing function at the input and calculating the resulting response, again at the input. The stress required to maintain a constant rate of strain will, except for transient terms, be the integral over the line of the viscous term alone.

This gives a viscosity equal to $r l^{3.4}=\eta\left(M_{c}\right)\left(\frac{M}{M_{c}}\right)^{3.4}$.

The steady-state response to sinusoidal stress or strain can be found almost as easily. This is the

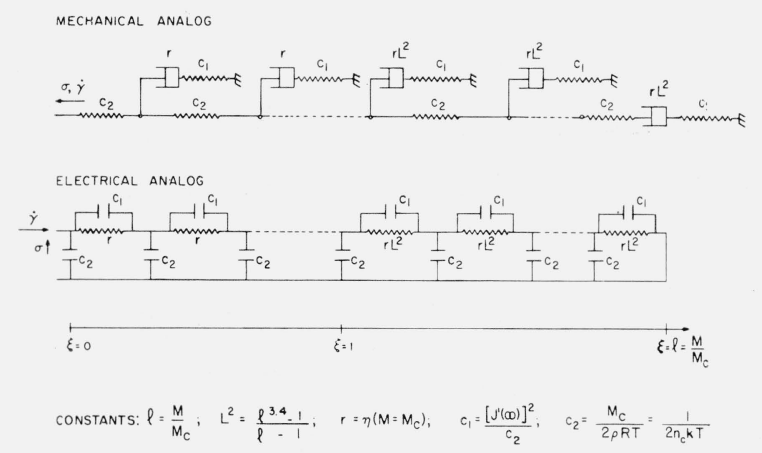

FIGURE 3. Model including effects of entanglements. problem of determining the input impedance of a two section electrical transmission line. From the differential equations relating the shear stress $\sigma$ to the shear strain $\gamma$ :

$$
\begin{aligned}
\dot{\gamma}_{\xi} & =-y \sigma \\
\sigma_{\xi} & =-z \dot{\gamma}
\end{aligned}
$$

where

$$
\begin{aligned}
\sigma_{\xi} & =\frac{\partial \sigma}{\partial \xi} \\
\dot{\gamma} & =\frac{\partial \gamma}{\partial t} \\
y & =i \omega c_{2} \\
z & =\frac{r}{1+i \omega c_{1} r} \quad 0 \leq \xi \leq 1 \\
& =\frac{r L^{2}}{1+i \omega c_{1} r L^{2}} \quad 1<\xi \leq l
\end{aligned}
$$

we derive :

$$
\dot{\gamma}_{\xi \xi}=y z \dot{\gamma}
$$

For steady-state sinusoidal stress and strain both force and response must be proportional to $\exp (i \omega t)$. Inserting this, plus the boundary conditions that $\sigma(l)=0$ and that both stress and strain must be continuous at $\xi=1$ we obtain:

$$
\begin{aligned}
G^{*}(i \omega) & =\frac{1}{J^{*}(i \omega)}=\frac{\sigma(\eta)}{\gamma(U)} \\
& =\frac{\Gamma_{1}}{c_{2}} \cdot \frac{\tanh \Gamma_{1}+\rho \tanh \Gamma_{3}}{1+\rho \tanh \Gamma_{1} \tanh \Gamma_{3}}
\end{aligned}
$$

where

$$
\begin{aligned}
\Gamma_{1} & =\sqrt{\frac{i \omega c_{2} r}{1+i \omega c_{1} r}} \\
\Gamma_{2} & =\sqrt{\frac{i \omega c_{2} r L^{2}}{1+i \omega c_{1} r L^{2}}} \\
\Gamma_{3} & =\frac{K}{L} \Gamma_{2}, \quad K=L(l-1) \\
\rho & =\frac{\Gamma_{2}}{\Gamma_{1}} .
\end{aligned}
$$

These expressions can be simplified immediately because of the fact that $c_{2}>>c_{1}$ and $c_{1} r L^{2}<<1$ for all cases of interest. Because of these relations, the denominators of $\Gamma_{1}, \Gamma_{2}$, and $\Gamma_{3}$ can be taken as unity for all frequencies up to those for which the hyperbolic tangents become essentially unity. Thus, for real frequencies, eq (3.1) becomes: 


$$
\begin{aligned}
G^{*}(i \omega) & =\frac{\alpha}{c_{2} \sqrt{1+\frac{c_{1}}{c_{2}} \alpha^{2}}} \frac{\tanh \alpha+L \tanh K \alpha}{1+L \tanh \alpha \tanh K \alpha} \\
= & \frac{\alpha}{c_{2} \sqrt{1+\frac{c_{1}}{c_{2}} \alpha^{2}}} \\
& \times \frac{(L+1) \sinh (K+1) \alpha+(L-1) \sinh (K-1) \alpha}{(L+1) \cosh (K+1) \alpha-(L-1) \cosh (K-1) \alpha},
\end{aligned}
$$

$$
\alpha=\sqrt{i \omega c_{2} r} \text {. }
$$

For our later extension of this expression into the complex plane to derive expressions for the transient response of the model, we shall have to go back to the full expression (3.1).

Equation (3.2) can be coded for machine computation in complex form, but it is still rather complicated for algebraic analysis. The relative values of the constants required to represent the behavior of a typical rubberlike polymer suggest several approximations, valid in different frequency ranges.

At very low frequencies the hyperbolic tangents can be expanded in power series, yielding for the low frequency limits $\left(\omega c_{2} r K^{2}<\frac{\pi^{2}}{4}\right)$ :

$$
\begin{aligned}
G^{*}(i \omega)= & G^{\prime}(\omega)+i G^{\prime \prime}(\omega) \\
= & \frac{\omega^{2} c_{2} r^{2}}{3}\left(L K^{3}+3 L^{2} K^{2}+3 L K+1\right)+\ldots \\
& +i[\omega r(L K+1)+\ldots] \\
& \approx \frac{\omega^{2} r^{2} c_{2}}{3} l^{6.8}(l+2)+i \omega r l^{3.4}+\ldots
\end{aligned}
$$$$
\text { (for } l^{3.4}>>1 \text { ). }
$$

$$
\begin{aligned}
J^{*}(i \omega)=J^{\prime}(\omega)-i J^{\prime \prime}(\omega) & \\
= & \frac{c_{2}}{3} \frac{L K^{3}+3 L^{2} K^{2}+3 L K+1}{(L K+1)^{2}}+\ldots \\
& -i\left[\frac{1}{\omega r(L K+1)}+\ldots\right] \\
& \approx \frac{c_{2}}{3}(l+2)-\frac{i}{\omega r l^{3.4}}+\ldots \quad\left(\text { for } l^{3.4}>>1\right) .
\end{aligned}
$$

When $\omega$ becomes large enough so that tanh $K \alpha$, but not $\tanh \alpha$, is essentially unity, eq (3.2) reduces to:

$$
\begin{aligned}
G^{*}(i \omega) & =\frac{\alpha}{c_{2}} \cdot \frac{\tanh \alpha+L}{1+L \tanh \alpha} \\
& =\frac{\alpha}{c_{2}} \operatorname{ctnh}(\alpha+\chi) ; \tanh \chi \equiv \frac{1}{L}
\end{aligned}
$$

since $\sqrt{1+i \omega c_{1} r}$ can still be taken as essentially unity. This can be separated into real and imaginary parts giving:

$$
\begin{aligned}
& G^{\prime}(\omega)=\frac{\theta}{c_{2}} \cdot \frac{\sinh 2(\theta+\chi)+\sin 2 \theta}{\cosh 2(\theta+\chi)-\cos 2 \theta} \\
& G^{\prime \prime}(\omega)=\frac{\theta}{c_{2}} \cdot \frac{\sinh 2(\theta+\chi)-\sin 2 \theta}{\cosh 2(\theta+\chi)-\cos 2 \theta} \\
& J^{\prime}(\omega)=\frac{c_{2}}{2 \theta} \cdot \frac{\sinh 2(\theta+\chi)+\sin 2 \theta}{\cosh 2(\theta+\chi)+\cos 2 \theta} \\
& J^{\prime \prime}(\omega)=\frac{c_{2}}{2 \theta} \cdot \frac{\sinh 2(\theta+\chi)-\sin 2 \theta}{\cosh 2(\theta+\chi)+\cos 2 \theta}
\end{aligned}
$$

where

$$
\begin{aligned}
& \theta=\sqrt{\frac{\omega c_{2} r}{2}}=\frac{\alpha}{1+i}, \\
& \text { and } \frac{25}{c_{2} r K^{2}}<\omega<\frac{25}{c_{2} r} .
\end{aligned}
$$

At still higher frequencies, both hyperbolic tangents can be taken as unity, but the denominator of $\Gamma_{1}$ must be retained, giving us:

$$
\begin{aligned}
& G^{*}=\frac{1}{\sqrt{c_{1} c_{2}}} \cdot \frac{1}{\sqrt{1-\frac{i}{\omega c_{1} r}}} \\
& \left.G^{\prime \prime},\right\}=\frac{1}{\sqrt{2 c_{1} c_{2}}} \frac{\left[\sqrt{1+\frac{1}{\left(\omega c_{1} r\right)^{2}}} \pm 1\right.}{\sqrt{1+\left(\frac{1}{\omega c_{1} r}\right)^{2}}} \\
& \left.\begin{array}{l}
J^{\prime} \\
J^{\prime \prime}
\end{array}\right\}=\sqrt{\frac{c_{1} c_{2}}{2}}\left[\sqrt{1+\left(\frac{1}{\omega c_{1} r}\right)^{2}} \pm 1\right]^{1 / 2} \text {, for } \omega \geq \frac{25}{c_{2} r} .
\end{aligned}
$$

The expressions for the imaginary parts of the modulus and compliance can be differentiated explicitly and equated to zero to find the extrema of these curves. However, the resulting transcendental equations are too complicated to be solved other than numerically, so this procedure is not of much value. Certain approximate expressions for the extrema can be found, based on the fact that the functions are closely approximated by regions proportional to some power of frequency, with only narrow intermediate frequency regions.

The imaginary part of eq (3.6) can be approximated by three expressions, approximately valid in different frequency regions, and from the intersection of these expressions approximate values for the maximum and minimum in $G^{\prime \prime}$ obtained. In the region where both $\theta$ and $\chi$ must be considered, we obtain two approximations. 
For $\theta<\chi$ we find: $G^{\prime \prime} \approx \frac{\theta}{c_{2} \chi}$

$$
\text { For } \theta>\chi \quad: G^{\prime \prime} \approx \frac{\chi}{2 c_{2} \theta} \text {. }
$$

The intersection of these two curves, at $\theta^{2}=\frac{1}{2} \chi^{2}$ or $\omega=\frac{1}{c_{2} r L^{2}}$, locates approximately the low-frequency maximum in $G^{\prime \prime}$.

When the frequency rises so that $\chi$ can be neglected compared to $\theta$, we predict $G^{\prime \prime}=\frac{\theta}{4}$, and the intersection of this with the second of the preceding two gives for a minimum:

$$
\theta^{2} \approx \frac{2}{L} \quad \text { or } \omega \approx \frac{4}{c_{2} r L} .
$$

These expressions are fairly good approximations for the frequencies of the extrema of the calculated curves shown in figure 4. As might be expected, the corresponding predictions of the magnitude of $G^{\prime \prime}$ at its extrema are not particularly good.

By a similar procedure, the minimum and maximum of $J^{\prime \prime}$ are predicted. The frequency of the minimum in $J^{\prime \prime}$ is approximately

$$
\theta \approx\left(\frac{3}{4} \chi\right)^{1 / 3} \quad \text { or } \omega \approx \frac{2}{c_{2} r}\left(\frac{3}{4 L}\right)^{2 / 3},
$$

that of the maximum

$$
\theta \approx \frac{\left(3 c_{2}\right)^{1 / 3}}{2} \quad \text { or } \omega \approx \frac{\left(3 c_{2}\right)^{2 / 3}}{2 c_{2} r} .
$$

The high frequency maximum in $G^{\prime \prime}$ can be obtained by the explicit differentiation of $G^{\prime \prime}$ in (3.8), yielding: $\omega=\frac{1}{\sqrt{3} c_{1} r}$.

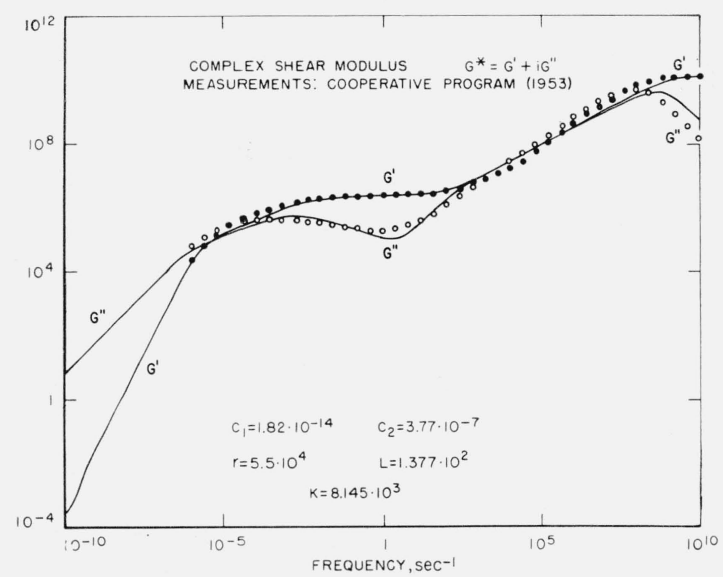

Figure 4.-Comparison of theory with experiment.

Complex modulus, polyisobutylene. Lines, this theory; points, measurements

\section{Transient Response}

\subsection{Theoretical Considerations}

The transient functions, $G(t)$ the stress relaxation function, and $J(t)$ the creep function, are given by $[6]$ :

$$
\begin{aligned}
& G(t)=(1 / 2 \pi i) \int_{c-i \infty}^{c+i \infty}\left(G^{*}(p) / p\right) \exp (p t) d p \\
& J(t)=(1 / 2 \pi i) \int_{c-i \infty}^{c+i \infty}\left(J^{*}(p) / p\right) \exp (p t) d p
\end{aligned}
$$

where $G^{*}(p)$ and $J^{*}(p)$ are the steady-state functions of section 3 continued into the complex frequency plane with $p=s+i \omega$. It is convenient for subsequent use to write them in the form:

$$
\begin{aligned}
G^{*}(p) & =1 / J^{*}(p) \\
& =\frac{\Gamma_{1}}{c_{2}} \frac{(\rho+1) \sinh \left(\Gamma_{3}+\Gamma_{1}\right)+(\rho-1) \sinh \left(\Gamma_{3}-\Gamma_{1}\right)}{(\rho+1) \cosh \left(\Gamma_{3}+\Gamma_{1}\right)-(\rho-1) \cosh \left(\Gamma_{3}-\Gamma_{1}\right)}
\end{aligned}
$$

with

$$
\begin{aligned}
\Gamma_{1}=\sqrt{\frac{c_{2}}{c_{1}}} \sqrt{\frac{p c_{1} r}{1+p c_{1} r}}, \\
\Gamma_{2}=\sqrt{\frac{c_{2}}{c_{1}}} \sqrt{\frac{p c_{1} r L^{2}}{1+p c_{1} r L^{2}}}, \Gamma_{3}=\frac{K}{L} \Gamma_{2}, \rho=\frac{\Gamma_{2}}{\Gamma_{1}} .
\end{aligned}
$$

The functions $G^{*}(p) / p$ and $J^{*}(p) / p$ are both analytic throughout the complex plane except for certain singularities on the negative real axis, including one for $J^{*}(p) / p$ at the origin, which is a second order pole. The others are first order poles except for two accumulation points at

$$
p^{\prime}=-1 /\left(c_{1} r\right) \text { and } p^{\prime \prime}=-1 /\left(c_{1} r L^{2}\right)
$$

which are essentially singular. In both cases the simple poles occur in two infinite sets, one between 0 and $p^{\prime \prime}$, the other between $p^{\prime \prime}$ and $p^{\prime}$.

Both functions are single valued and both are even functions of $\Gamma_{1}$ and $\Gamma_{2}$. For this reason we are at liberty to choose either sign for the radicals, and we shall choose it to yield positive real parts for $\Gamma_{1}$ and $\Gamma_{2}$.

No singularities occur to the left of $p^{\prime}$. The paths of integration can thus be deformed to a finite circle in the left half plane enclosing all poles except that at the origin for $J^{*}(p) / p$. This yields the following a priori bounds for $G(t)$ and $J(t)$ :

$$
\begin{aligned}
& G(t) \mid \leq M_{1} \\
& J(t) \mid \leq M_{2}(1+t), M_{1} \text { and } M_{2} \text { constants, }
\end{aligned}
$$

for all $t \geq 0$. The term linear in $t$ comes from the second order pole at the origin.

Formal solutions of (4.1) and (4.2) valid for all times may be written as: 


$$
\begin{gathered}
G(t)=\sum_{1}^{\infty} g_{n} \exp \left(p_{n} t\right) \\
J(t)=\frac{t}{r(1+L K)}+\frac{c_{2}}{1+L K}\left[L K+\frac{1+L K^{3}}{3(1+L K)}\right] \\
-\sum_{1}^{\infty} j_{m} \exp \left(p_{m} t\right)
\end{gathered}
$$

where the poles $p_{n}$ and $p_{m}$ are the roots of:

$\left(\rho_{n}+1\right) \cosh \left(\Gamma_{3, n}+\Gamma_{1, n}\right)$

$$
-\left(\rho_{n}-1\right) \cosh \left(\Gamma_{3, n}-\Gamma_{1, n}\right)=0
$$

$\left(\rho_{m}+1\right) \sinh \left(\Gamma_{3, m}+\Gamma_{1, m}\right)$

$$
+\left(\rho_{m}-1\right) \sinh \left(\Gamma_{3, m}-\Gamma_{1, m}\right)=0 .
$$

From the relations $G(0)=G^{*}(\infty)$ and $J(0)=J^{*}(\infty)$ [9] we conclude:

$$
\begin{gathered}
\sum_{1}^{\infty} g_{n}=\left(c_{1} c_{2}\right)^{-1 / 2} \\
\sum_{1}^{\infty} j_{m}=\frac{c_{2}}{1+L K}\left[L K+\frac{1+L K^{3}}{3(1+L K)}\right]-\left(c_{1} c_{2}\right)^{1 / 2} .
\end{gathered}
$$

\subsection{Expressions for the Long Time Range, $t \geq 10^{-4} c_{2} r(K+1)^{2}$}

For the very long time region the equations for the poles and corresponding expressions for the residues can be written as:

$(L+1) \cos (K+1) \tau_{n}-(L-1) \cos (K-1) \tau_{n}=0$

$$
g_{n}=\frac{2 / c_{2}}{1+(K / L)\left[1+\left(L^{2}-1\right) \sin ^{2} \tau_{n}\right]}, \tau_{n}=\left(-p_{n} c_{2} r\right)^{1 / 2}
$$

and $G(t) \approx \sum_{1}^{N} g_{n} \exp \left(-\tau_{n}^{2} t / c_{2} r\right)$.

$(L+1) \sin (K+1) \mu_{m}+(L-1) \sin (K-1) \mu_{m}=0$,

$$
\mu_{m}=\left(-p_{m} c_{2} r\right)^{1 / 2}
$$

$$
j_{m}=\frac{2 c_{2} / \mu_{m}^{2}}{1+(K / L)\left[1+\left(L^{2}-1\right) \cos ^{2} \mu_{m}\right]}
$$

and $\sum_{1}^{\infty} j_{m} \exp \left(p_{m} t\right) \approx \sum_{1}^{N} j_{m} \exp \left(-\mu_{m}^{2} t / c_{2} r\right)$

It is readily seen that $\tau_{n}$ and $\mu_{m}$ are bounded respectively by $\left(n-\frac{1}{2} \pm \frac{1}{2}\right) \pi /(K+1)$ and $\left(m \pm \frac{1}{2}\right) \pi /(K+1)$.

For the residues we have the bounds:

$$
\begin{gathered}
(1+K L)^{-1} \leq c_{2} g_{n} / 2 \leq(1+K / L)^{-1} \\
(K+1)^{2}\left(m+\frac{1}{2}\right)^{-2} \pi^{-2}(1+K L)^{-1} \\
\leq \frac{j_{m}}{2 c_{2}} \leq(K+1)^{2}\left(m-\frac{1}{2}\right)^{-2} \pi^{-2}(1+K / L)^{-1} .
\end{gathered}
$$

These series are asymptotic in time, and in both cases the error involved in stopping at the $N$ th term will be less than 0.1 percent for

$$
t \geq c_{2} \gamma(K+1)^{2} N^{-2} .
$$

Our neglect of $c_{1}$ in this region is justified since the value of $p_{N} c_{1} r L^{2}$ will be no greater than $(N+1)^{2} \pi^{2}\left(c_{1} / c_{2}\right) L^{2}(K+1)^{-2}$, which is small for at least the first several hundred terms. In practice it is not feasible to compute more than 100 to 200 poles and residues. Hence we turn to other methods for shorter times.

\subsection{Representation for the Short and Medium Time Ranges, $t \leq 10^{-4} c_{2} r(K+1)^{2}$}

The series of the preceding section correspond to transforms of the Mittag-Leffler expansions of $G^{*}(p) / p$ and $J^{*}(p) / p$. For shorter times we have to find expansions which are centered around infinity. Such expansions can be found in the form of exponential series in powers of $\exp \left(-2 \Gamma_{1}\right)$ and $\exp \left(-2 \Gamma_{3}\right)$. For convenience of notation we write them as Dirichlet series by introducing the definitions:

$$
x_{1}=\exp \left(-2 \Gamma_{1}\right), x_{3}=\exp \left(-2 \Gamma_{3}\right), \zeta=(\rho-1) /(\rho+1) .
$$

We find then:

$$
\begin{gathered}
\frac{G^{*}(p)}{p}=\frac{\Gamma_{1}}{c_{2} p} \frac{1+\zeta x_{1}}{1-\zeta x_{1}} \frac{1-x_{3} \frac{\zeta+x_{1}}{1+\zeta x_{1}}}{1-x_{3} \frac{\zeta-x_{1}}{1-\zeta x_{1}}} \\
\frac{J^{*}(p)}{p}=\frac{c_{2}}{\Gamma_{1} p} \frac{1-\zeta x_{1}}{1+\zeta x_{1}} \frac{1-x_{3} \frac{\zeta-x_{1}}{1-\zeta x_{1}}}{1-x_{3} \frac{\zeta+x_{1}}{1+\zeta x_{1}}}
\end{gathered}
$$

It can be shown that $\zeta, x_{1}$, and $x_{3}$ are less than 1 in absolute value for all points off the negative real $p$-axis. Hence there will be a region around the negative real axis outside of which $\frac{\zeta \pm x_{1}}{1 \pm \zeta x_{1}} x_{3}$ will be less than one in absolute value. The following expansions hold:

$$
\begin{aligned}
\frac{G^{*}(p)}{p}= & \frac{\Gamma_{1}}{c_{2} p}\left\{1+2 \sum_{1}^{\infty}\left(\zeta x_{1}\right)^{\nu}\right\} \\
& \cdot\left\{1-2 x_{1} x_{3} \frac{1-\zeta^{2}}{1-\zeta^{2} x_{1}^{2}} \sum_{0}^{\infty}\left(\frac{\zeta-x_{1}}{1-\zeta x_{1}} x_{3}\right)^{\nu}\right\}
\end{aligned}
$$




$$
\begin{aligned}
\frac{J^{*}(p)}{p} & =\frac{c_{2}}{\Gamma_{1} p}\left\{1+2 \sum_{1}^{\infty}\left(-\zeta x_{1}\right)^{\nu}\right\} \\
& \times\left\{1+2 x_{1} x_{3} \frac{1-\zeta^{2}}{1-\zeta^{2} x_{1}^{2}} \sum_{0}^{\infty}\left(\frac{\zeta+x_{1}}{1+\zeta x_{1}} x_{3}\right)^{\nu}\right\} .
\end{aligned}
$$

The series are asymptotic in both $x_{1}$ and $x_{3}$, i.e., if we terminate after $n$ terms in $x_{3}$ the remainder is $0\left(x_{3}{ }^{n+1}\right)$. If we replace the second brackets of (4.12) and (4.13) merely by $1+0\left(x_{3}\right)$, it is not useful to go beyond the number of terms of the $x_{1}$ expansion which results in a remainder term of $0\left(x_{3}\right)$.

We can estimate the error involved in truncating the $x_{1}$ series by means of the following theorem [10]:

Theorem: If $L(x)$ is a function, continuous and positive on the real interval $0 \leq x \leq x_{0}$, with the property

$$
\lim _{x \rightarrow 0} L(u x) / L(x)=1 \text { for all fixed } u>0,
$$

then if $\mathscr{L}(F)==f(p)$, with real $F(t)$, is convergent for $0 \leq p \leq p_{0}$ and $f(p)$ has the asymptotic behavior

$$
f(p) \sim\left(C / p^{\alpha}\right) L(1 / p) \text { for } p \rightarrow \infty, \quad C>0, \quad \alpha>0,
$$

and if $F(t) \geq 0$, then

$$
\int_{0}^{t} F(t) d t \sim \frac{C}{\Gamma(\alpha+1)} t^{\alpha} L(t) \quad \text { for } t \rightarrow 0 .
$$

Furthermore, if $F(t)$ is monotonic in the neighborhood of $t=0$, differentiation is allowed to yield the asymptotic behavior of $F(t)$ itself.

This theorem can be used to estimate the truncation error if we apply the inverse Laplace transform to a finite number of terms in eqs (4.12) and (4.13). We shall use it only to determine the range of usefulness of the leading terms, since these are in fact the only ones which can be transformed in closed form. Putting them on the left-hand side, we write:

$$
\begin{gathered}
G(t)-G^{0}(t) \sim \mathscr{L}^{-1}\left\{\frac{\Gamma_{1}}{c_{2} p} 2 \sum_{1}^{M}\left(\zeta x_{1}\right)^{\nu}+0\left(x_{3}\right)\right\} \\
\sim \mathscr{L}^{-1}\left\{\frac{2 \Gamma_{1}}{c_{2} p} \zeta x_{1}\right\} \\
J^{0}(t)-J(t) \sim \mathscr{L}^{-1}\left\{-\frac{c_{2}}{\Gamma_{1} p} 2 \sum_{1}^{\infty}\left(-\zeta x_{1}\right)^{\nu}+0\left(x_{3}\right)\right\} \\
\sim \mathscr{L}^{-1}\left\{\frac{2 c_{n}}{\Gamma_{1} p} \zeta x_{1}\right\} .
\end{gathered}
$$

The expressions on the right under the $\mathscr{L}^{-1}$ signs satisfy the conditions of the theorem. After differentiation we get the following remainder terms:

$$
\begin{aligned}
G(t) & -G^{0}(t) \\
\sim \frac{1}{\sqrt{c_{1} c_{2}}} \frac{L^{2}-1}{L^{2}} \frac{t}{c_{1} r} & \exp \left(-2 \sqrt{\frac{\overline{c_{2}}}{c_{1}}}\left(1+\frac{t}{c_{1} r}\right)^{-1 / 2}\right) \\
& \left(1+\frac{1}{2} \sqrt{\frac{\overline{c_{2}}}{c_{1}}} \frac{\left(t / c_{1} r\right)}{\left(1+\frac{t}{c_{1} r}\right)^{3 / 2}}\right)
\end{aligned}
$$

$J^{0}(t)-J(t)$

$$
\begin{gathered}
\sim \sqrt{c_{1} c_{2}} \frac{L^{2}-1}{L^{2}} \frac{t}{c_{1} r} \exp \left(-2 \sqrt{\frac{\underline{c}_{2}}{c_{1}}}\left(1+\frac{t}{c_{1} r}\right)^{-1 / 2}\right) \\
\left(1+\frac{1}{2} \sqrt{\frac{c_{2}}{c_{1}}} \frac{\left(t / c_{1} r\right)}{\left(1+\frac{t}{c_{1} r}\right)^{3 / 2}}\right) .
\end{gathered}
$$

$G^{0}(t)$ and $J^{0}(t)$, the inverse transforms of the leading terms of eqs (3.12) and (3.13), are tabulated functions:

$$
G^{0}(t)=\left(c_{1} c_{2}\right)^{-1 / 2} \exp \left(-t / 2 c_{1} r\right) I_{0}\left(t / 2 c_{1} r\right)
$$

$$
\begin{aligned}
J^{0}(t)=\left(c_{1} c_{2}\right)^{1 / 2} \exp \left(-t / 2 c_{1} r\right) & {\left[\left(1+t / c_{1} r\right) I_{0}\left(t / 2 c_{1} r\right)\right.} \\
& \left.+\left(t / c_{1} r\right) I_{1}\left(\frac{t}{2 c_{1} r}\right)\right]
\end{aligned}
$$

where $I_{0}(x)$ and $I_{1}(x)$ are modified Bessel functions. For very short times these may be expanded in power series, giving:

$$
\begin{array}{r}
G^{0}(t)=\frac{1}{\sqrt{c_{1} c_{2}}}\left[1+\sum_{1}^{\infty} \frac{(-1)^{\nu}(2 \nu) !}{(\nu !)^{3}}\left(\frac{t}{4 c_{1} r}\right)^{\nu}\right] \\
J^{0}(t)=\sqrt{c_{1} c_{2}}\left[1+\sum_{1}^{\infty} \frac{(-1)^{\nu+1}(2 \nu) !}{(\nu !)^{3}(2 \nu-1)}\left(\frac{t}{4 c_{1} r}\right)^{\nu}\right] .
\end{array}
$$

These expansions are useful in the range $0 \leq t \leq 10 c_{1} r$. For longer times we can employ asymptotic expansions of the Bessel functions which yield:

$$
\begin{aligned}
& G^{0}(t) \sim \sqrt{\frac{r}{\pi c_{2} t}}\left[1+\frac{c_{1} r}{4 t}+\ldots\right] \\
& J^{0}(t) \sim 2 \sqrt{\frac{c_{2} t}{\pi r}}\left[1-\frac{c_{1} r}{4 t}+\ldots\right]
\end{aligned}
$$

We will be in error by less than 0.1 percent by taking $G(t)=G^{\circ}(t)$ for times less than $c_{2} r / 182$, and $J(t)=J^{\circ}(t)$ for times less than $c_{2} r / 49$. We still need an expression to cover the range

$$
c_{2} r / 182 \leq t \leq 10^{-4} c_{2} r(K+1)^{2},
$$

the upper limit being that established for the practical use of the residue series. If we set $c_{1}=0$ in eqs (4.12) and (4.13) we obtain series which can be transposed term by term, giving: 


$$
G(t)=\sqrt{\frac{r}{c_{2} \pi t}}\left[1+2 \sum_{1}^{M}\left(\frac{L-1}{L+1}\right)^{\nu} \exp \left(-\frac{\nu^{2} c_{2} r}{t}\right)\right]
$$

$$
\begin{aligned}
J(t)=2 & \sqrt{\frac{c_{\ominus} t}{\pi r}}\left[1+2 \sum_{1}^{M}\left(-\frac{L-1}{L+1}\right)^{\nu}\right. \\
& \left.\left\{\exp \left(-\frac{\nu^{2} c_{2} r}{t}\right)-\nu \operatorname{erfc}\left(\nu \sqrt{\frac{c_{2} r}{t}}\right)\right\}\right]
\end{aligned}
$$

where $M \leq[K]$, that is we may not carry these series beyond $[K]$ terms in view of our neglect of the $x_{3}$ series $\left(x_{3}=x_{1}^{K}\right.$ for $\left.c_{1}=0\right)$.

The essential singularity at $p^{\prime \prime}$ precludes any rigorous calculation of a remainder term associated with the neglect of $c_{1}$ in this region of time. Widder's formula [11] gives a series of approximations of order $N$ for $G(t)$ in terms of $G^{*}(p)$ and its first $N$ derivatives evaluated at $p=N / t$. For any finite approximation, the terms in $c_{1}$ vanish with increasing $t$, but the expression becomes exact only in the limit of $N \rightarrow \infty$, and no estimate is available for the error associated with the approximation involved in stopping with some finite $N$.

A more convincing rationale for the neglect of $c_{1}$ in this region can probably be based on physical considerations. $c_{1}$ was introduced into the model to account for the glassy compliance, which must be added to the rubberlike compliance but is significant only at very short times or high frequencies. The steady-state functions change, in a fairly narrow frequency range, from the region where $G^{\prime} \propto \omega^{1} / 2$ and $J^{\prime} \propto \omega^{-1} / 2$, characteristic of the model with $c_{1}=0$, to their limiting values of $\left(c_{2} c_{1}\right)^{-1 / 2}$ and $\left(c_{2} c_{1}\right)^{1 / 2}$. At lower frequencies $c_{1}$ does not appear in either $G^{*}(i \omega)$ or $J^{*}(i \omega)$. Since our expressions for $G^{\circ}(t)$ and $J^{\circ}(t)$ are valid into the region of time where they are proportional to $t^{-1 / 2}$ and $t^{1 / 2}$, corresponding to the transforms of $p^{1 / 2}$ and $p^{-1 / 2}$, we should be quite justified in using the simpler model with $c_{1}=0$ at longer times.

In practice we may need up to 250 terms in eqs (4.23) and (4.24), not a difficult task for a modern computer. We have purposely set the limits so that more terms are required here than in the residue series, since it is easier to compute the exponential and error functions than to determine the poles from the transcendental eqs (4.8) and (4.9).

\section{A Trial of the Theory}

To test the predictions of the theory, we utilize the experimental results available on the NBS "standard" polyisobutylene. ${ }^{3}$ Although these results are rather old, they still comprise the most complete set of data available for any single polymer. Direct measurements are available covering essentially the whole response curve for the steady state and stress relaxation functions, and creep measurements for times greater than and equal to that corresponding to the pseudo-equilibrium plateau.

\footnotetext{
3 "Standard" here refers to a particular batch of polymer from which samples
were distributed to permit comparison of different measurement techniques and results as described in reference 12 . This comparison was concluded several years results as described in reference 12 . This com
ago, and no further samples are available.
}

The dynamic measurements used are those obtained by several investigators as summarized by Marvin [12]. The stress relaxation measurements are those reported by Tobolsky and Catsiff [13], converted from Young's to shear modulus by the use of a factor $1 / 3$ throughout. Although this factor is not strictly correct near the glassy modulus, the error is not serious since the shear modulus apparently never exceeds $1 / 4$ of the bulk modulus. The creep data are unpublished results of Leaderman and Overberg using a concentric cylinder viscometer [14].

The constants needed can be obtained from the empirical expressions for the viscosity of polyisobutylene given by Fox et al. [7]. Values of $r$ and $c_{2}$ were calculated from the critical molecular weight and the viscosity of polyisobutylene at this molecular weight. The measured viscosity and molecular weight of the "standard" polyisobutylene are not consistent with the expressions given by Fox, probably because the milling required to obtain a large uniform batch produced a larger than normal low molecular weight fraction. Thus, for consistent results, we must either use a fictitious molecular weight corresponding to the measured viscosity or modify the constants of Fox's empirical relations.

The best results are obtained by the use of a fictitious molecular weight of $1.03 \times 10^{6}$, corresponding to the measured viscosity of the polymer, rather than the value of $1.56 \times 10^{6}$ from light scattering or even that of $1.35 \times 10^{6}$ from intrinsic viscosity measurements. In a previous publication [6] the results of this same theory for the dynamic functions only were presented with $l$ calculated from the relation $\eta=r l^{3.5}$, the ezponent corresponding to Bueche's theoretical prediction [8]. Here we use $\eta=r l^{3.4}$, corresponding to the empirical relation. The difference in the calculated results is small, but those obtained here using the 3.4 power agree slightly better with the experiments than did the former calculations

The constants used, calculated for a temperature of $25^{\circ} \mathrm{C}$, and their source, are summarized as follows: $c_{2}=M_{c} /(2 \rho R T)=3.77 \times 10^{-7} . \quad M_{c}$ from Fox et al. [7] $r=5.5 \times 10^{4}$. Derived from the viscosity of polyisobutylene with molecular weight of $M_{c}$ [7] $l=(\eta / r)^{1 / 3.4}=60.12, \quad \eta$ the measured viscosity at $25^{\circ} \mathrm{C}=10^{10.79}$.

$$
L=\left[\frac{l 3^{4}-1}{l-1}\right]^{1 / 2}=1.377 \times 10^{2}
$$

$K=L(l-1)=8.145 \times 10^{3}$

$c_{1}=\left(J^{\prime}(\infty)\right)^{2} / c^{2}=1.82 \times 10^{-14}$, using the value $J^{\prime}(\infty)$ from the dynamic measurements.

The results are presented in figures $4,5,6$, and 7 , where the lines represent values calculated from the theory and the points the experimental measurements. The agreement is striking in view of the wide range of values covered. All the features of the experimental curves are reproduced qualitatively, with the changes in the character of the curves occurring at nearly the correct frequencies or times. The maximum deviation between the calculated 


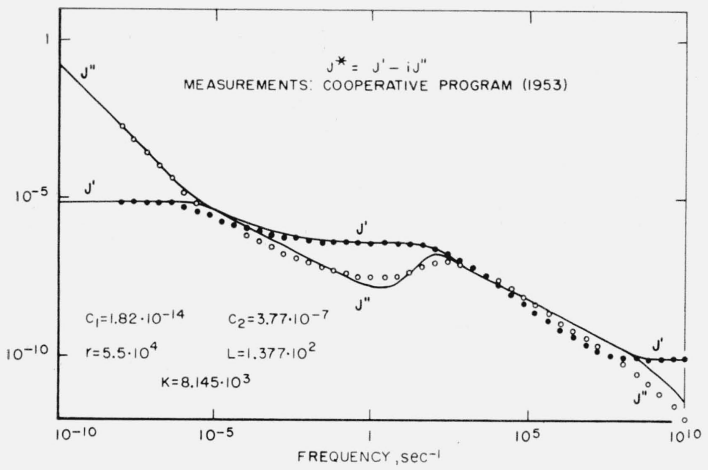

Figure 5. Comparison of theory with experiment. Complex compliance.

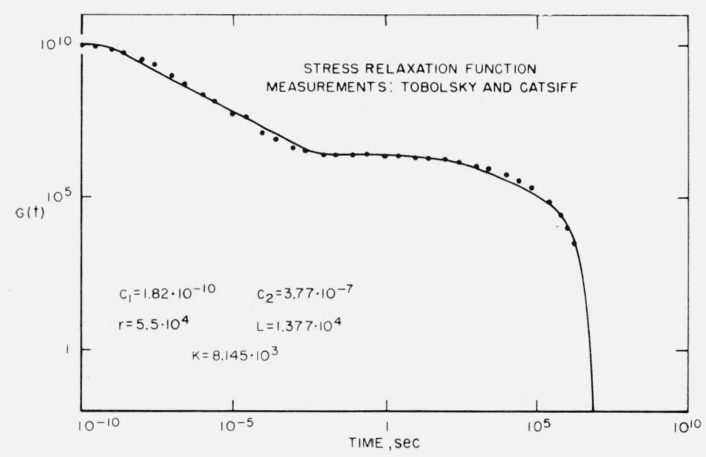

Figure 6. Comparison of theory with experiment. Stress relaxation.

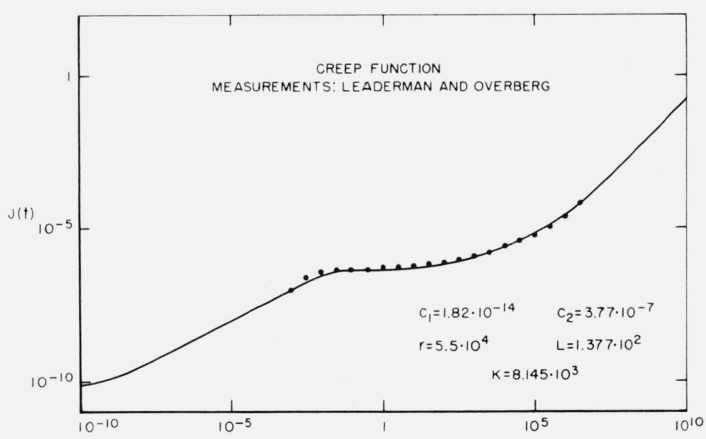

Figure 7. Comparison of theory with experiment. Creep.

and experimental values is about 0.06 on a logarithmic scale, corresponding to about a 15 percent difference, except for a narrow region of frequency for $J^{\prime \prime}$ and $G^{\prime \prime}$, where the difference reaches about 0.3 logarithmically. The real parts of the dynamic functions and the transient functions agree more closely with the measured values. A result we did not expect is that the transient curves appear to be represented by the theory somewhat better than $G^{\prime}$ and $J^{\prime}$.

The theory is certainly not exact. In particular, the prediction of a proportionality to the square root of time or frequency (or its inverse) in the region between the rubbery and glassy plateaus is only approximated by the measured results.

However, the theory yields quite satisfactory results. They certainly agree with the measurements as closely as most calculations comparing different types of measurements through the use of approximation methods now available. It is particularly interesting to note that the values of both the pseudoequilibrium plateau and the equilibrium compliance agree with the measurements to well within the experimental uncertainties. These values as calculated by the theory, it should be recalled, depend on constants obtained entirely from steady flow viscosity measurements. It is thus quite apparent that the same "entanglements", whatever their true nature, are responsible for the break in the viscositymolecular weight relation and thepseudo-equilibrium rubbery plateau, as assumed in the theory.

The necessity, in these calculations for the "standard" polyisobutylene, of using a fictitious molecular weight precludes any chance of using these results to check on the effect of molecular weight distribution. This question must remain in abeyance pending calculations for other systems, preferably fractionated polymers.

The major part of the work involved in developing the transient expressions was financed by the Office of Naval Research under Contract No. NR384-310. During the preparation of the final manuscript coauthor R. S. Marvin was Visiting Professor of the Department of Polymer Chemistry of Kyoto University, Kyoto, Japan. The facilities for study and research provided by Kyoto University are gratefully acknowledged.

\section{References}

[1] P. E. Rouse, Jr., J. Chem. Phys. 21, 1272 (1953).

[2] F. Bueche, J. Chem. Phys. 22, 603 (1954).

[3] B. H. Zimm, J. Chem. Phys. 24, 269 (1956).

[4] J. D. Ferry, R. F. Landel, and M. L. Williams, J. Appl. Phys. 26, 359 (1955); F. Bueche, J. Appl. Phys. 26, 738 (1955).

[5] J. D. Ferry, Viscoelastic Properties of Polymers, ch. 10 (Wiley \& Sons, Inc., New York, N.Y., 1961).

[6] R. S. Marvin, Chapter in J. T. Bergen (Ed.), Viscoelasticity: Phenomenological Aspects (Academic Press, New York, N.Y., 1960).

[7] T. G Fox, S. Gratch, and S. Loshaek, Chapter in F. Eirich (Ed.), Rheology, Theory and Applications, Vol. I (Academic Press, New York, N.Y., 1956).

[8] F. Bueche, J. Chem. Phys. 20, 1959 (1952); ibid. 25, 599 (1956).

[9] B. Van der Pol and H. Bremmer, Operational Calculus Based on the Two-Sided Laplace Integral, VII, 4 (Cambridge University Press, 1955).

[10] G. Doetsch, Handbuch der Laplace Transformation, Vol. 1 (Verlag Birkhäuser, Basel, 1950).

[11] Ref. [9], formula VII-58.

[12] R. S. Marvin, Proc. 2nd International Congress Rheology, Ed. by V. G. Harrison (Butterworth, London, 1953).

[13] A. V. Tobolsky and E. Catsiff, J. Poly. Sci. 19, 111 (1956).

[14] H. Leaderman and R. J. Overberg (unpublished results).

(Paper 66B4-85) 